\title{
Evolution of Dark Matter Halo Density Profiles and Substructure from $\Lambda$ CDM Simulations
}

\author{
Darren Reed, ${ }^{1,2} \dagger$ Fabio Governato, ${ }^{1,3}$ Licia Verde, $, 4,5$ Jeffrey Gardner,${ }^{6}$ \\ Thomas Quinn, ${ }^{1}$ Joachim Stadel, ${ }^{7}$ David Merritt, ${ }^{8}$ and George Lake ${ }^{9}$ \\ ${ }^{1}$ ICC, Dept. of Physics, Univ. of Durham, Rochester Building, Science Laboratories, South \\ Road, Durham DH1 3LE, UK \\ ${ }^{2}$ Astronomy Dept., Univ. of Washington, Box 351580, Seattle, WA 98195 USA \\ ${ }^{3}$ INAF, Osservatorio Astronomico di Brera, via Brera 28, I-20131 Milano, Italy \\ ${ }^{4}$ Dept. of Physics \& Astronomy, Univ. of Pennsylvania, 209 South 33rd Street, Philadelphia, \\ PA 19104-6396, USA \\ ${ }^{5}$ Dept. of Astrophysical Sciences, Princeton Univ., Peyton Hall, Ivy Lane, Princeton, NJ 08544 \\ USA \\ ${ }^{6}$ Pittsburgh Supercomputing Center, 4400 Fifth Avenue, Pittsburgh, PA 15213, USA \\ ${ }^{7}$ Inst. for Theoretical Physics, University of Zurich, Winterthurerstrasse 190, 8057, Switzerland \\ ${ }^{8}$ Dept. of Physics, Rochester Inst. of Technology, 84 Lomb Memorial Dr., Rochester, NY \\ 14623-5603, USA \\ ${ }^{9}$ Dept. of Physics, PO Box 642814, Pullman, WA 99164 USA
}

\begin{abstract}
We use $\Lambda \mathrm{CDM}$ numerical simulations to model the density profiles and substructure populations in a set of sixteen dark matter halos with resolutions of up to seven million particles within the virial radius. These simulations allow us to follow robustly the formation and evolution of the central cusp over a large mass range of $10^{11}$ to $10^{14} \mathrm{M}_{\odot}$ down to approximately $0.5 \%$ of the virial radius, and from redshift 5 to the present. The cusp of the density profile is set at redshifts of two or greater and remains remarkably stable to the present time, when considered in non-comoving coordinates.

We fit our halos to a 2 parameter profile where the steepness of the asymptotic cusp is given by $\gamma$, and its radial extent is described by the concentration, $c_{\gamma}$. In our simulations, we find $\gamma$ $=1.4-0.08 \log \left(M / M_{*}\right)$ for halos of $0.01 M_{*}$ to $1000 M_{*}$, with a large scatter of $\Delta \gamma \sim \pm 0.3$; and $c_{\gamma}=8\left(M / M_{*}\right)^{-0.15}$ with a large $M / M_{*}$ dependent scatter roughly equal to $\pm c_{\gamma}$. Our redshift zero halos have inner slope parameters ranging approximately from $\mathrm{r}^{-1}$ to $\mathrm{r}^{-1.5}$, with a median of roughly $\mathrm{r}^{-1.3}$. This two parameter profile fit works well for all halo types present in our simulations, whether or not they show evidence of a steep asymptotic cusp.

The substructure population is independent of host halo mass and redshift with halo to halo scatter in the substructure velocity distribution function of a factor of roughly two to four. The radial distribution of substructure halos (subhalos) is consistent with the mass profile over the radial range where the possibility of artificial numerical disruption of subhalos can be most reliably excluded, $\mathrm{r} \gtrsim 0.3 \mathrm{r}_{v i r}$, although a weakly shallower subhalo profile is favored by the data. We discuss the implications that our results have on gravitational lensing studies of halo structure.
\end{abstract}

\section{Introduction}

The mass distribution of dark matter halos provides a direct probe of the nature of the dark matter particle. The inner structure of dark matter halos and the distribution of "subhalos" within dark matter halos are particularly sensitive to dark matter properties,

$\dagger$ Email: d.s.reed@durham.ac.uk. 
and provide a critical test of the $\Lambda$ CDM model. Strong gravitational lensing in clusters can potentially provide a direct measurement of the halo mass profile, and indeed central mass profiles for several lensing clusters have been calculated (Tyson, Kochanski, \& Dell'Antonio 1998; Shapiro \& Iliev 2000; Sand, Treu, \& Ellis 2002; Gavazzi et al. 2003; Sand et al. 2004), but have yielded sometimes conflicting results. Gravitational lensing can also be used to infer the substructure population, even when no luminous counterparts are present (e.g. Dalal \& Kochanek 2002; Metcalf et al. 2004). Although any effects that baryons may have on observable characteristics and potentially on the dark matter distribution complicates conclusions derived from comparisons with dark matter simulations, it is still necessary to first understand the properties of the dark matter alone. In this work, we analyse the density profiles and substructure population of $16 \Lambda \mathrm{CDM}$ simulated halos covering three decades in mass, from dwarfs to clusters, each with $\sim 10^{6}$ or more particles.

\section{The Simulations}

We use the parallel gravity solver PKDGRAV (Stadel 2001) for all of our numerical simulations, which model a $\Lambda$ CDM cosmology with $\Omega_{m}=0.3$ and $\Lambda=0.7$, and are consistent with WMAP measurements (e.g. , Bennett et al. 2003; Spergel et al. 2003). The runs are described in detail in Reed et al. 2003ab, 2004)

Our sample includes ten clusters extracted from one cosmological volume of uniform resolution (CUBEHI), allowing us to study cosmological variance. For the six simulations of individual halos, we use a "renormalized volume" technique of nested resolutions, where the halo of interest is modelled at high resolution within a low resolution cosmological environment (e.g. Katz \& White 1993; Ghigna et al. 1998). Our two highest resolution runs are a seven million particle group and a four million particle cluster Some of our halos are well-resolved to redshifts of three or higher, allowing investigation of mass or redshift-dependent trends.

We perform a convergence study using lower resolution versions of a volume-renormalized galaxy, group, and cluster. From these, we find that density profiles are robust down to $\mathrm{r}_{\min }=\mathrm{N}_{\mathrm{p}}^{-1 / 3}$. These runs also verify that the minimum subhalo circular velocity down to which our results are complete, $\mathrm{v}_{\mathrm{c}, \mathrm{lim}}$, is robust. We only follow the evolution of halos to redshifts where their particle number exceeds $10^{5}$ for density profiles and $3.5 \times 10^{5}$ for subhalos. This allows us to avoid problems associated with numerical discreteness effects, which tend to lower the lower the cusp densities through two-body interactions (see e.g. Diemand et al. 2004). Subhalos are particularly sensitive to numerical problems because subhalos with numerically softened cusps are more easily disrupted, especially near host halo centres where tidal forces are large.

\section{Density Profiles}

The range in asymptotic-fit cusp slope of our density profiles is bounded approximately by $\mathrm{r}^{-1}$ (as in Navarro, Frenk, \& White 1997; NFW hereafter) and $\mathrm{r}^{-1.5}$ (as in Moore et al. 1999); see Fig. 1. None of halos have slopes shallower than $\mathrm{r}^{-1}$ down to $\mathrm{r}_{\min }$. Most of the halo slopes continue to get shallower down to $r_{\min }$ while a few of them show signs of having reached an asymptotic slope. We find that our density profiles are well fit by a two parameter equation of the form:

$$
\rho=\frac{\rho_{s}}{\left(c_{\gamma} r / r_{\text {vir }}\right)^{\gamma}\left[1+\left(c_{\gamma} r / r_{\text {vir }}\right)\right]^{3-\gamma}},
$$




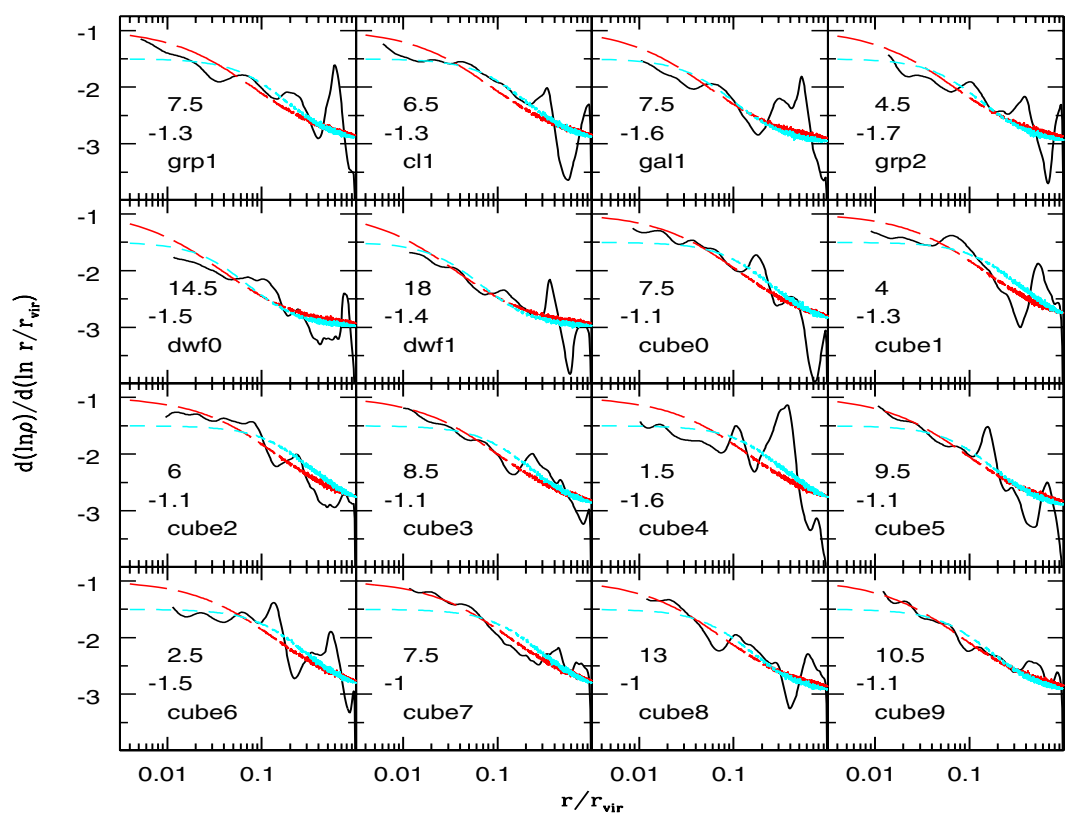

Figure 1. Density profile slopes of our 16 halos sample at $z=0$ shown by solid curves (black). The long dashed curve (red) shows the results of best-fit concentration to the NFW profile $\left(\rho_{\text {cusp }} \propto \mathrm{r}^{-1}\right)$. Short-dashes (blue) show the best-fit Moore et al. profile $\left(\rho_{\text {cusp }} \propto r^{-1.5}\right)$. Results of a two parameter fit where both the concentration, $\mathrm{c}_{\gamma}$ (top number) and the asymptotic cusp slope parameter, $(-) \gamma$ (bottom number), are shown in each plot window.

where $\gamma$ is the asymptotic inner slope parameter $\left(\rho_{\text {cusp }} \alpha \mathrm{r}^{-\gamma}\right)$, and $c_{\gamma}$ is the concentration parameter $\left(c_{\gamma}=r_{v i r} / r_{s}\right.$, where $r_{\mathrm{s}}$ is the scale radius $)$.

Figure 2 shows evidence of a trend in $\gamma$ with $M / M_{*}$ :

$$
\gamma \simeq 1.4-0.08 \log _{10}\left(M / M_{*}\right)
$$

with a scatter of $\Delta \gamma \sim \pm 0.3$ for our halos, and valid for halos of $0.01 M_{*}$ to $1000 M_{*}$. $\mathrm{M}_{*}$ is the characteristic mass of collapsing halos defined by the scale at which the rms linear density fluctuation equals the threshold for non-linear collapse (i.e. $\sigma\left(M_{*}(z)\right)=\delta_{c}$ ). Halo concentration also shows a trend with $M / M_{*}$ given by:

$$
c_{\gamma} \simeq 8\left(M / M_{*}\right)^{-0.15},
$$

with a $M / M_{*}$ dependent scatter roughly equal to $\pm c_{\gamma} \cdot \dagger$

The trends can be qualitatively explained by a simple spherical infall halo formation model. Small $M / M_{*}$ halos form early, when the mean and virial density of the universe is changing rapidly, which gives them smaller and steeper cusps. We also find evidence that for halos of similar mass, the cusps are assembled earliest in halos where they are steepest, and that halos with the highest concentrations form earliest, though our statistical sample of ten clusters from the uniform resolution cube is small. Note that once the cusp material is assembled at $\mathrm{z} \gtrsim 2$, the non-comoving (physical) profile shape shows remarkably little evolution.

$\dagger$ Code to reproduce this profile for halos of user chosen mass and redshift can be downloaded from http://www.icc.dur.ac.uk/ reed/profile.html. 


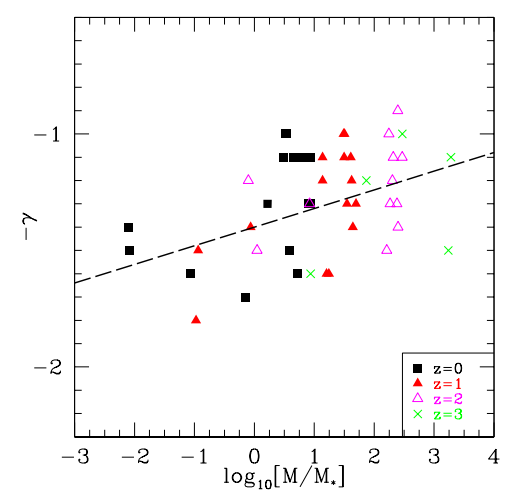

(a)

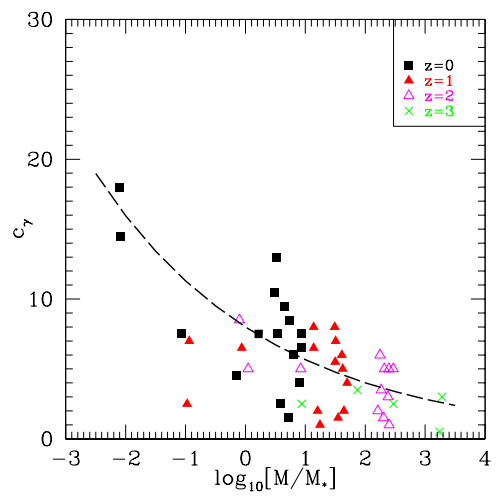

(b)

Figure 2. a) The value of the inner slope parameter (panel a) and the concentration parameter (panel b) for halos over a wide range of masses and redshifts. Dashed curves show fits to these halos as given by Eqn. 3.2 and 3.3.

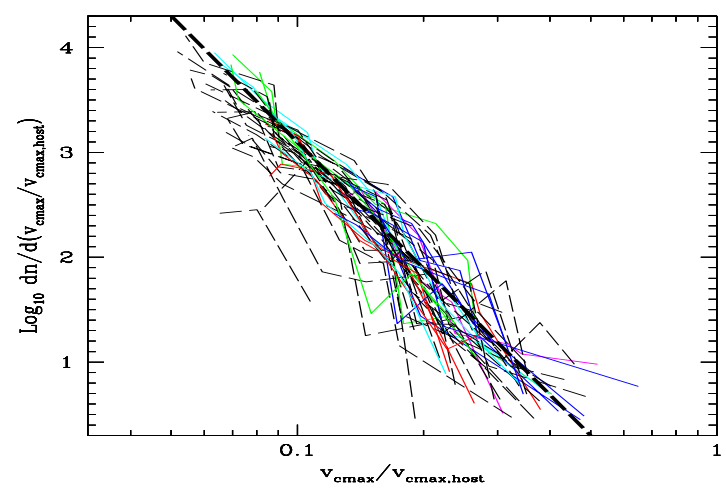

Figure 3. Subhalo VDF for all halos with $\mathrm{N}_{\mathrm{p}}>3.5 \times 10^{5}$ at redshifts $0,0.5,1$, and 2, normalized to $\mathrm{v}_{\mathrm{cmax}}$, host and a virial volume of unity. Solid lines (colours) are renormalized volumes; dashed lines (black) are the ten clusters in the CUBEHI simulation. Heavy dashed line corresponds to Eqn. 4.1.

\section{Substructure}

Subhalos are selected using SKID (Stadel et al. 2001). The subhalo population is generally self-similar (Fig. 3). The subhalo velocity distribution function (VDF) follows the relation

$$
\mathrm{dn} / \mathrm{dv}=\frac{1}{8}\left(\frac{\mathrm{v}_{\mathrm{cmax}}}{\mathrm{v}_{\mathrm{cmax}, \text { host }}}\right)^{-4},
$$

over our sampled range of $0.07 \mathrm{v}_{\mathrm{cmax}}$, host to $0.4 \mathrm{v}_{\mathrm{cmax}}$, host with roughly a factor of 2 to 4 in halo to halo scatter. When we extend to our highest resolution halos, we still find weak or no redshift evolution in the VDF of individual halos to redshift 4.

The radial distribution of the subhalo population, shown for GRP1 in Fig. 4, has a slope equal to or slightly shallower than the slope of the density profile for each resolution except at small radii ( $\lesssim 0.2-0.3 \mathrm{r}_{\text {vir }}$ ), where subhalos are highly deficient. At larger radii, a subhalo radial distribution that is shallower than the mass profile is favoured in our data, and is also reported by e.g. Diemand, Moore \& Stadel (2004), Gao et al. (2004). 


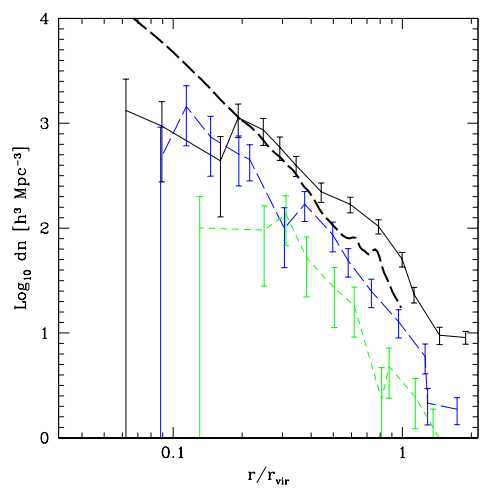

(a)

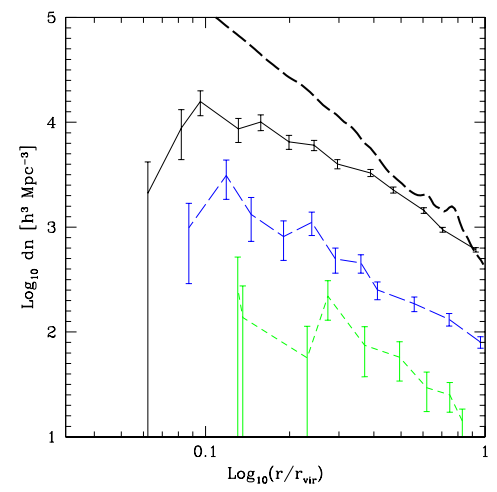

(b)

Figure 4. The radial distribution of subhalos in GRP1 for each of the 3 different resolutions, plotted down to the minimum complete circular velocity $\mathrm{v}_{\mathrm{c}, \mathrm{lim}}$ (panel a), and plotted down to the $\mathrm{N}_{\mathrm{p} \text {, subhalo }}=32$ particles (panel b). $1 \sigma$ poisson error bars are shown. The dashed line is the particle distribution. Note that the relative normalisations are arbitrary since the different resolutions are each plotted down to their separate resolution-dependent $\mathrm{v}_{\mathrm{c}, \text { lim }}$ or mass.

However, a radial subhalo slope equal to the mass profile slope is not ruled out except in the central region, where artificial numerical effects may dominate. Given the uncertainties, a larger sample of higher resolution halos is needed to determine the minimum converged radius of the subhalo radial distribution.

The anti-bias at small radii is likely to be a result of increased tidal stripping near the larger central tidal field. Simulations are not able to follow subhalos once they lose enough mass to drop below the resolution limits. Supporting this, our median subhalo masses are smaller at smaller host radii, indicative of tidal stripping. Note that there is a much stronger anti-bias if subhalos are selected by mass instead of circular velocity. This is because the subhalo $\mathrm{v}_{\mathrm{c} \text {,max }}$ has a relatively weak mass dependence. Because of this, the median median subhalo $\mathrm{v}_{\mathrm{c}, \text { max }}$ has weak or no dependence on host-centric radius. Additionally, there is a radial trend in subhalo internal angular momenta (the 'spin' parameter) of smaller spins at smaller radii. This is likely to be caused by stripping of high angular momentum material from the outer parts of large spin subhalos.

\section{Power Law Cosmologies, $\mathbf{P} \alpha \mathbf{k}^{\mathrm{n}}$}

We have modelled our renormalized cluster simulation in cosmologies where the spectral index of primordial of initial density fluctuations, $n$, is fixed over all scales with $\sigma_{8}=1.0$. We have simulated four values of $n(0,-1,-2,-2.7)$. We find that smaller values of $n$ yield shallower density cusps with less substructure, with values of $\alpha=1.1$ and $\mathrm{c}_{\gamma}=5.5$ for $n=-2.7$, and $\alpha=1.5$ and $c_{\gamma}=14$ for $n=0$. Note that the $n$ dependence on profile shapes would imply shallower profiles for low mass $\Lambda$ CDM halos, which is opposite to the trend in our $\Lambda \mathrm{CDM}$ halos, indicating that any profile dependence on $n$ is outweighed by the $M / M_{*}$ dependence.

\section{Conclusions}

Density profiles from our simulations are robust down to less than $1 \% \mathrm{r}_{\mathrm{vir}}$, enabling comparisons with lensing results over similar scales. However, because the central regions 
of clusters are baryon dominated (e.g. Sand et al. 2004), it is not clear how directly comparable cusp slopes derived from lensing studies are to dark matter simulations.

Halo substructure radial profiles are roughly consistent with the halo density profile for radii greater than $\sim 0.3 \mathrm{r}_{\text {vir }}$, although a weakly shallower subhalo distribution is favored by the simulations. Higher resolution simulations are needed to conclusively determine how much, if any of the stronger anti-bias at $\lesssim 0.1-0.3 \mathrm{r}_{\mathrm{vir}}$ is due to artificial numerical disruption. Taylor \& Babul (these proceedings) discuss evidence for artificial bias in numerical simulations.

Substructure is a likely cause of the anomalous flux ratios seen in a number of lensing systems (e.g. Kochanek \& Dalal 2004). Spectroscopic lensing studies are able to probe galaxy substructure at mass scales of $\lesssim 10^{7} \mathrm{M}_{\odot}$ at small radii (Metcalf et al. 2004). However, simulations with mass resolution better than the current $\sim 10^{6-7}$ particles are needed to model $\Lambda \mathrm{CDM}$ substructure down to such small scales.

\section{Acknowledgements}

DR has been support PPARC and by the NASA Graduate Student Researchers Program. FG was partially supported by NSF grant AST-0098557 at the University of Washington. TRQ was partially supported by the National Science Foundation. Simulations were performed on the Origin 2000 at NCSA and NASA Ames, the IBM SP4 at the Arctic Region Supercomputing Center (ARSC) and at CINECA (Bologna, Italy), the NASA Goddard HP/Compaq SC 45, and at the Pittsburgh Supercomputing Center.

\section{References}

Bardeen, J.M., Bond, J.R., Kaiser, N., \& Szalay, A.S., 1986, ApJ, 305, 15.

Bennett, C. L. et al., 2003, ApJS, 148, 97B

Dalal N., \& Kochanek C. S., 2002, ApJ, 572, 25

Diemand J., Moore B., Stadel J., \& Kazantzidis S., 2004, MNRAS, 348, 977

Diemand J., Moore B., \& Stadel J., 2004, MNRAS, 352, 535

Gao L., De Lucia G., White S.D.M., \& Jenkins A., 2004, MNRAS, 352, L1

Gavazzi R., Fort B., Mellier Y., Pello R., \& Dantel-Fort M., 2003, A\&A, 403, 11

Ghigna S., Moore B., Governato F., Lake G., Quinn T., \& Stadel J., 1998, MNRAS, 300, 146

Hayashi E., et al., 2003, astro-ph/0310576

Katz N., \& White S., 1993, ApJ, 412, 478

Kochanek C.S., \& Dalal N., 2004, ApJ, 610, 69

Metcalf R.B., Moustakas L.A., Bunker A.J., \& Parry I.R., 2004, ApJ, 607, 43

Moore B., Quinn T., Governato F., Stadel J., \& Lake G., 1999 (M99), MNRAS, 310, 1147

Navarro J. F., Frenk C. S., \& White S. D. M., 1997, ApJ, 490, 493 (NFW)

Reed D., Gardner J., Quinn T., Stadel J., Fardal M., Lake G., \& Governato F., 2003a, MNRAS, 346,565

Reed D., Governato F., Verde L, Gardner J., Quinn T., Stadel J., Merritt D., \& Lake G., 2003b, astro-ph/0312544

Reed D., Governato F., Quinn T., Gardner J., Stadel J., \& Lake G., 2004, astro-ph/0406034

Sand D. J., Treu T., \& Ellis R. S., 2002, ApJ, 574, L129

Sand D. J., Treu T., Smith G. P., \& Ellis R. S., 2004, ApJ, 604,88

Shapiro P. R., \& Iliev I. T., 2000, ApJ, 542L, 1

Spergel D., et al., 2003, ApJS, 148, 175

Stadel, J., 2001, PhDT, SKID available at http://hpcc.astro.washington.edu.

Taylor J., \& Navarro J., 2001, ApJ, 563483

Tyson J. A., Kochanski G. P., \& Dell'Antontio I. P. 1998, ApJ, 498, L107 